\title{
THE PREPARATION AND CHARACTERIZATION OF SOME NOVEL FEROCENIL DERIVATIVES
}

\author{
DANIJELA ILIĆ KOMATINA ${ }^{1}$, ALEKSANDRA MINIĆ JANČIĆ', JOVANA BUGARINOVIĆ \\ DRAGANA STEVANOVIĆ ${ }^{2}$
}

${ }^{1}$ Faculty of Technical Sciences, University of Priština in Kosovska Mitrovica, Serbia

${ }^{2}$ Faculty of Science, University of Kragujevac, Kragujevac, Serbia

\begin{abstract}
This paper provides a report on an easily performable synthesis via one-pot reaction, between the newly synthesized 3-arylamino-1-ferrocenylpropan-1-ols and PhNCO. We have used the acetic acid as the catalyst and ultrasound irradiation as the reaction promoter. We have evaluated the reaction score on three examples. In order to gain a more comprehensive insight into the role of $\alpha$-ferrocenyl carbocation, we have described a plausible mechanism of the transformations in question. Moreover, we have purified the prepared by using column chromatography. Besides, we provide a detailed characterization of the new compounds (products and starting material), which was performed by IR, NMR and elemental analyses.
\end{abstract}

Keywords: Tetrahydropirimidine ring, Ferrocene, Intramolecular cyclization, $\alpha$-Ferrocenyl carbocation, Spectral characterization.

\section{INTRODUCTION}

Over the past few years, we have witnessed a radical change for modern organic chemistry, namely, raising awareness of the issues of environmental sustainability and efficiency. Consequently, one-pot reactions which involve various types of cascade and tandem reactions are being increasingly seen as a favourable approach due to their higher efficiency, operational simplicity, increased cost effectiveness and reduced generation of chemical waste. This is particularly striking in comparison with other synthetic methodologies (Hayashi 2016; Nicolaou et al., 2006; Motokura et al., 2005; Yang et al., 2016; Domling et al., 2012). In a similar vein, a new mainstream method has emerged, especially in the development of sustainable, frequently catalyst- or/and solvent-free versions of challenging reactions (Sarma et al., 2012a). Moreover, from the perspective of industrial manufacturing and "green" chemistry, such strategies would result in high yields of relatively pure products in shorter reaction times. Therefore, these strategies can be seen as not only eco-friendly, but also as financially viable options (Sharma et al., 2012b).

In contrast, heterocycles constitute one of the largest classes of organic compounds (Eicher et al., 2013). Heterocycles represent important structural motifs which are to be found extensively across a range of biologically active natural products, drug intermediates, pharmaceuticals and dye chemistry, as well as common versatile building blocks used for the synthesis of a variety of compounds (Taylor et al., 2016). For instance, at present, the most dominant among prescription medicaments constitute small heterocyclic molecules (containing diverse functional groups), or more complex structures containing a

*Coresponding author: danijela.ilic@pr.ac.rs heterocyclic core fragment (Taylor et al., 2016). Within the class of heterocycles, in recent years we have seen an increased interest of both chemists and biologists for urea derivatives, which have since been synthesized extensively. This specific class of compounds is characterized by a wide range of biological activities (Kashawa et al., 2009; Khan et al., 2008; Kaneko et al., 2007; Li et al., 2009; Tale et al., 2011; Fernandez \& Caballero, 2006), which consequently resulted in a heightened interest for their synthesis. For this reason, the synthesis and characterization of novel cyclic ureas bearing ferrocenyl group obtained in this manner could be increasingly significant in the future.

Our team have recently reported synthesis of bioactive 2-ferrocenoyl ethyl aryl amines (Damljanović et al., 2011; Pejović et al., 2012a). These results are a proof of our sustained interest in the synthesis of novel $\mathrm{Fc}$-containing $(\mathrm{Fc}=$ ferrocene $)$ heterocyclic compounds, which are of potential biological significance (Pejović et al., 2012b; Pejović et al., 2017; Pejović et al., 2018a; Pejović et al., 2018b), and in the design and optimizations of reactions conditions. 2-Ferrocenoyl ethyl aryl amines - Mannich bases have demonstrated great initial results in the synthesis of Fc derivatives (Minić et al., 2017; Minić et al., 2018; Minić et al., 2019), notably in the synthesis of sixmembered ureas (Minić et al., 2015; Minić et al., 2020a).

In view of this, we report the synthesis completed via onepot reaction, between novel 3-arylamino-1-ferrocenylpropan-1ols (which was obtained from corresponding Mannich bases), phenyl isocyanate, and acetic acid. All the above mentioned compounds used in this synthesis, including the products and the starting material, have been subjected to purification by column chromatography and their structure has been confirmed with the use of spectroscopic data $\left({ }^{1} \mathrm{H}-\mathrm{NMR},{ }^{13} \mathrm{C}-\mathrm{NMR}, 2 \mathrm{D}-\mathrm{NMR}\right.$ and IR). Moreover, elemental analyses have been used in this 
process. Furthermore, this synthetic approach generates novel Fc-containing heterocyclic systems which may prove to be biologically relevant.

\section{EXPERIMENTAL}

\section{Materials and measurements}

All chemicals were commercially available and used as received, except that the solvents were purified by distillation. Chromatographic separations were carried out using Silica Gel 60 (Merck, 230-400 mesh ASTM), whereas Silica Gel 60 on Al plates, layer thickness $0.2 \mathrm{~mm}$ (Merck) was used for TLC. Melting points were determined on a Mel-Temp capillary melting points apparatus, model 1001 and are uncorrected. Microanalysis of carbon and hydrogen were carried out with a Carlo Erba 1106 microanalyser; their results agreed favorably with the calculated values. IR measurements were carried out with a Perkin-Elmer FTIR 31725-X spectrophotometer. NMR spectra were recorded on a Bruker Avance III $400 \mathrm{MHz}\left({ }^{1} \mathrm{H}\right.$ at $400 \mathrm{MHz},{ }^{13} \mathrm{C}$ at $\left.101 \mathrm{MHz}\right)$ and Varian Gemini $\left({ }^{1} \mathrm{H}\right.$ at $200 \mathrm{MHz}$, ${ }^{13} \mathrm{C}$ at $\left.50 \mathrm{MHz}\right) \mathrm{NMR}$ spectrometers. Chemical shifts are reported in ppm $(\delta)$ values relative to TMS $\left(\delta_{\mathrm{H}} 0 \mathrm{ppm}\right)$ in ${ }^{1} \mathrm{H},{ }^{13} \mathrm{C}$ and heteronuclear 2D NMR spectra.

\section{Synthesis and spectral characterization}

General procedure for the synthesis of 3-(arylamino)-1ferrocenylproran-1-ones (3a-c)

Ketones 3a-c were synthesized by the known procedure (Pejović et al., 2012a; Minić et al., 2015; Minić et al., 2017; Minić et al., 2018; Minić et al., 2019). Spectral data of compounds 3a-c follow.

1-Ferrocenyl-3-((2-iodophenyl)amino)propan-1-one (3a). Dark red solid; mp $162{ }^{\circ} \mathrm{C}$. Yield 62\%. ${ }^{1} \mathrm{H}$ NMR $(400 \mathrm{MHz}$, $\left.\mathrm{CDCl}_{3}\right) \delta=7.71-7.67\left(\mathrm{~m}, 1 \mathrm{H}, \mathrm{CH}_{\mathrm{Ar}}\right), 7.30-7.25(\mathrm{~m}, 2 \mathrm{H}, 2 \times$ $\mathrm{CH}_{\mathrm{Ar}}$ ), $6.69\left(\mathrm{~d}, J=7.9 \mathrm{~Hz}, 1 \mathrm{H}, \mathrm{CH}_{\mathrm{Ar}}\right.$ ), 4.80 (pseudo t, $J=1.9 \mathrm{~Hz}$, $\left.2 \mathrm{H}, 2 \times \mathrm{CH}_{\mathrm{Cp}}\right), 4.68\left(\mathrm{t}, J=5.8 \mathrm{~Hz}, 1 \mathrm{H}, \mathrm{CH}_{2} \mathrm{CH}_{2} \mathrm{NH}\right), 4.54$ (pseudo t, $J=1.9 \mathrm{~Hz}, 2 \mathrm{H}, 2 \times \mathrm{CH}_{\mathrm{Cp}}$ ), $4.17\left(\mathrm{~s}, 5 \mathrm{H}, 5 \times \mathrm{CH}_{\mathrm{Cp}}\right.$ ), $3.65\left(\mathrm{q}, J=6.2 \mathrm{~Hz}, 2 \mathrm{H}, \mathrm{CH}_{2} \mathrm{C}_{2}{ }_{2} \mathrm{NH}\right), 3.11-3.05(\mathrm{~m}, 2 \mathrm{H}$, $\left.\mathrm{CH}_{2} \mathrm{CH}_{2} \mathrm{NH}\right) .{ }^{13} \mathrm{C} \mathrm{NMR}\left(101 \mathrm{MHz}, \mathrm{CDCl}_{3}\right) \delta=202.7(\mathrm{C}=\mathrm{O})$, $146.9\left(\mathrm{C}_{\mathrm{Ar}}\right), 139.3\left(\mathrm{C}_{\mathrm{Ar}}\right), 129.4\left(\mathrm{C}_{\mathrm{Ar}}\right), 118.8\left(\mathrm{C}_{\mathrm{Ar}}\right), 110.5\left(\mathrm{C}_{\mathrm{Ar}}\right)$, $85.8\left(\mathrm{C}_{\mathrm{Ar}}\right), 78.8\left(\mathrm{C}_{\mathrm{Cp}}\right), 72.5\left(2 \times \mathrm{C}_{\mathrm{Cp}}\right), 69.9\left(5 \times \mathrm{C}_{\mathrm{Cp}}\right), 69.3(2 \times$ $\left.\mathrm{C}_{\mathrm{Cp}}\right), 38.8\left(\mathrm{CH}_{2} \underline{\mathrm{CH}}_{2} \mathrm{NH}\right), 38.2\left(\underline{\mathrm{CH}}_{2} \mathrm{CH}_{2} \mathrm{NH}\right) . \mathrm{IR}\left(\mathrm{ATR}, \mathrm{cm}^{-1}\right): v$ $=3210(\mathrm{~N}-\mathrm{H}) \mathrm{cm}^{-1} ; \mathrm{v}=1651(\mathrm{C}=\mathrm{O}) \mathrm{cm}^{-1}$. Anal. Calc. for $\mathrm{C}_{19} \mathrm{H}_{18}$ FeINO: C, 49.71; H, 3.95; Fe, 12.16; I, 27.64; N, 3.05; O, 3.48. Found: C, 49.76, H, 3.91, N, 3.12\%.

1-Ferrocenyl-3-((3-iodophenyl)amino)propan-1-one (3b). Dark red solid; mp $156{ }^{\circ} \mathrm{C}$. Yield $74 \% .{ }^{1} \mathrm{H}$ NMR $(400 \mathrm{MHz}$, $\left.\mathrm{CDCl}_{3}\right) \delta=7.06-7.00\left(\mathrm{~m}, 2 \mathrm{H}, 2 \times \mathrm{CH}_{\mathrm{Ar}}\right), 6.94-6.85(\mathrm{~m}, 1 \mathrm{H}$, $\mathrm{CH}_{\mathrm{Ar}}$ ), $6.65-6.60\left(\mathrm{~m}, 1 \mathrm{H}, \mathrm{CH}_{\mathrm{Ar}}\right.$ ), 4.81 (pseudo t, $J=1.9 \mathrm{~Hz}$, $2 \mathrm{H}, 2 \times \mathrm{CH}_{\mathrm{Cp}}$ ), 4.54 (pseudo t, $J=1.9 \mathrm{~Hz}, 2 \mathrm{H}, 2 \times \mathrm{CH}_{\mathrm{Cp}}$ ), 4.30 $\left(\mathrm{s}, 1 \mathrm{H}, \mathrm{CH}_{2} \mathrm{CH}_{2} \mathrm{~N}\right.$ ) $, 4.15\left(\mathrm{~s}, 5 \mathrm{H}, 5 \times \mathrm{CH}_{\mathrm{Cp}}\right), 3.55(\mathrm{~s}, 2 \mathrm{H}$,
$\left.\mathrm{CH}_{2} \mathrm{CH}_{2} \mathrm{NH}\right), 3.03\left(\mathrm{t}, J=6.0 \mathrm{~Hz}, 2 \mathrm{H}, \mathrm{CH}_{2} \mathrm{CH}_{2} \mathrm{NH}\right) .{ }^{13} \mathrm{C} \mathrm{NMR}$ $\left(101 \mathrm{MHz}, \mathrm{CDCl}_{3}\right) \delta=203.3(\mathrm{C}=\mathrm{O}), 149.0\left(\mathrm{C}_{\mathrm{Ar}}\right), 130.8\left(\mathrm{C}_{\mathrm{Ar}}\right)$, $126.4\left(\mathrm{C}_{\mathrm{Ar}}\right), 121.3\left(\mathrm{C}_{\mathrm{Ar}}\right), 112.6\left(\mathrm{C}_{\mathrm{Ar}}\right), 95.4\left(\mathrm{C}_{\mathrm{Ar}}\right), 78.7\left(\mathrm{C}_{\mathrm{Cp}}\right), 72.5$ $\left(2 \times \mathrm{C}_{\mathrm{Cp}}\right), 69.9\left(5 \times \mathrm{C}_{\mathrm{Cp}}\right), 69.3\left(2 \times \mathrm{C}_{\mathrm{Cp}}\right), 38.5\left(\mathrm{CH}_{2} \mathrm{CH}_{2} \mathrm{NH}\right)$, $37.9\left(\mathrm{CH}_{2} \mathrm{CH}_{2} \mathrm{NH}\right)$. IR (ATR, $\left.\mathrm{cm}^{-1}\right): v=3230(\mathrm{~N}-\mathrm{H}) \mathrm{cm}^{-1} ; v=$ $1663(\mathrm{C}=\mathrm{O}) \mathrm{cm}^{-1}$. Anal. Calc. for $\mathrm{C}_{19} \mathrm{H}_{18}$ FeINO: C, 49.71; $\mathrm{H}$, 3.95; Fe, 12.16; I, 27.64; N, 3.05; O, 3.48. Found: C, 49.76, H, $3.99, \mathrm{~N}, 3.10 \%$.

1-Ferrocenyl-3-((4-iodophenyl)amino)propan-1-one (3c). Dark red solid; mp $160{ }^{\circ} \mathrm{C}$. Yield 56\%. ${ }^{1} \mathrm{H}$ NMR $(400 \mathrm{MHz}$, $\left.\mathrm{CDCl}_{3}\right) \delta=7.54-7.37\left(\mathrm{~m}, 2 \mathrm{H}, 2 \times \mathrm{CH}_{\mathrm{Ar}}\right), 6.58-6.36(\mathrm{~m}, 2 \mathrm{H}, 2$ $\times \mathrm{CH}_{\mathrm{Ar}}$ ), 4.77 (pseudo t, $J=2.0 \mathrm{~Hz}, 2 \mathrm{H}, 2 \times \mathrm{CH}_{\mathrm{Cp}}$ ), 4.54 (pseudo $\left.\mathrm{t}, J=2.0 \mathrm{~Hz}, 2 \mathrm{H}, 2 \times \mathrm{CH}_{\mathrm{Cp}}\right), 4.29\left(\mathrm{~s}, 1 \mathrm{H}, \mathrm{CH}_{2} \mathrm{CH}_{2} \mathrm{NH}\right), 4.16(\mathrm{~s}$, $\left.5 \mathrm{H}, 5 \times \mathrm{CH}_{\mathrm{Cp}}\right), 3.56\left(\mathrm{~s}, 2 \mathrm{H}, \mathrm{CH}_{2} \underline{\mathrm{C}}_{2} \mathrm{NH}\right), 3.01(\mathrm{t}, J=5.9 \mathrm{~Hz}, 2 \mathrm{H}$, $\left.\mathrm{C}_{2} \mathrm{CH}_{2} \mathrm{NH}\right) .{ }^{13} \mathrm{C} \mathrm{NMR}\left(101 \mathrm{MHz}, \mathrm{CDCl}_{3}\right) \delta=203.3(\mathrm{C}=\mathrm{O})$, $147.3\left(\mathrm{C}_{\mathrm{Ar}}\right), 137.9\left(\mathrm{C}_{\mathrm{Ar}}\right), 115.2\left(\mathrm{C}_{\mathrm{Ar}}\right), 78.7\left(\mathrm{C}_{\mathrm{Ar}}\right), 78.0\left(\mathrm{C}_{\mathrm{Cp}}\right), 72.6$ $\left(2 \times \mathrm{C}_{\mathrm{Cp}}\right), 69.9\left(5 \times \mathrm{C}_{\mathrm{Cp}}\right), 69.3\left(2 \times \mathrm{C}_{\mathrm{Cp}}\right), 38.6\left(\mathrm{CH}_{2} \mathrm{CH}_{2} \mathrm{NH}\right)$, $38.0\left(\underline{\mathrm{CH}}_{2} \mathrm{CH}_{2} \mathrm{NH}\right)$. IR (ATR, $\left.\mathrm{cm}^{-1}\right): v=3190(\mathrm{~N}-\mathrm{H}) \mathrm{cm}^{-1} ; v=$ $1657(\mathrm{C}=\mathrm{O}) \mathrm{cm}^{-1}$. Anal. Calc. for $\mathrm{C}_{19} \mathrm{H}_{18} \mathrm{FeINO}$ : C, 49.71; $\mathrm{H}$, 3.95; Fe, 12.16; I, 27.64; N, 3.05; O, 3.48. Found: C, 49.71, H, $3.93, \mathrm{~N}, 3.12 \%$.

General procedure for the synthesis of 3-(arylamino)-1ferrocenylproran-1-ols (4a-c)

Alcohols 4a-c were synthesized by the known procedure (Minić et al., 2015; Minić et al., 2017; Minić et al., 2018; Minić et al., 2019). Spectral data of compounds 4a-c follow.

1-Ferrocenyl-3-((2-iodophenyl)amino)propan-1-ol (4a). Yellow oil. Yield 97\%. ${ }^{1} \mathrm{H}$ NMR $\left(200 \mathrm{MHz}, \mathrm{CDCl}_{3}\right) \delta=7.66-$ $7.62\left(\mathrm{~m}, 1 \mathrm{H}, \mathrm{CH}_{\mathrm{Ar}}\right), 7.24-7.11\left(\mathrm{~m}, 1 \mathrm{H}, \mathrm{CH}_{\mathrm{Ar}}\right), 6.57(\mathrm{t}, J=8.8$ $\mathrm{Hz}, 1 \mathrm{H}, \mathrm{CH}_{\mathrm{Ar}}$ ), $6.47-6.36\left(\mathrm{~m}, 1 \mathrm{H}, \mathrm{CH}_{\mathrm{Ar}}\right.$ ), 4.52 (pseudo $\mathrm{t}, J=$ $\left.1.9 \mathrm{~Hz}, 2 \mathrm{H}, 2 \times \mathrm{CH}_{\mathrm{Cp}}\right), 4.28-4.21\left(\mathrm{~m}, 1 \mathrm{H}, \mathrm{C}^{\mathrm{HOHCH}} \mathrm{CH}_{2} \mathrm{NH}\right)$, 4.17 (s, $7 \mathrm{H}, 7 \times \mathrm{CH}_{\mathrm{Cp}}$ *overlapped signals), $3.30-3.27(\mathrm{~m}, 2 \mathrm{H}$, $\left.\mathrm{CHOHCH}_{2} \underline{\mathrm{C}}_{2} \mathrm{NH}\right), 2.24-2.12\left(\mathrm{~m}, 1 \mathrm{H}, \mathrm{CHOHCH}_{2} \mathrm{CH}_{2} \mathrm{NH}\right)$, $2.05-2.01\left(\mathrm{~m}, 2 \mathrm{H}, \mathrm{CHOHC}_{2} \mathrm{CH}_{2} \mathrm{NH}\right) .{ }^{13} \mathrm{C} \mathrm{NMR}(50 \mathrm{MHz}$, $\left.\mathrm{CDCl}_{3}\right) \delta=147.3\left(\mathrm{C}_{\mathrm{Ar}}\right), 138.9\left(\mathrm{C}_{\mathrm{Ar}}\right), 129.3\left(\mathrm{C}_{\mathrm{Ar}}\right), 118.4\left(\mathrm{C}_{\mathrm{Ar}}\right)$, $110.5\left(\mathrm{C}_{\mathrm{Ar}}\right), 93.3\left(\mathrm{C}_{\mathrm{Ar}}\right), 85.4\left(\mathrm{C}_{\mathrm{Cp}}\right), 68.3(\underline{\mathrm{CHOH}}), 68.0\left(5 \times \mathrm{C}_{\mathrm{Cp}}\right)$, $67.9\left(\mathrm{C}_{\mathrm{Cp}}\right), \quad 66.7 \quad\left(\mathrm{C}_{\mathrm{Cp}}\right), \quad 65.5 \quad\left(\mathrm{C}_{\mathrm{Cp}}\right), \quad 65.4 \quad\left(\mathrm{C}_{\mathrm{Cp}}\right), \quad 41.4$ $\left(\mathrm{CHOHCH}_{2} \mathrm{CH}_{2} \mathrm{NH}\right), 36.7\left(\mathrm{CHOHCH}_{2} \mathrm{CH}_{2} \mathrm{NH}\right)$. IR $\left(\mathrm{ATR}, \mathrm{cm}^{-1}\right)$ : $v=3280(\mathrm{~N}-\mathrm{H}) \mathrm{cm}^{-1} ; v=3074(\mathrm{O}-\mathrm{H}) \mathrm{cm}^{-1}$. Anal. Calc. for $\mathrm{C}_{19} \mathrm{H}_{20}$ FeINO: C, 49.49; H, 4.37; Fe, 12.11; I, 27.52; N, 3.04; O, 3.47. Found: C, 49.52, H, 4.31, N, 3.10\%.

1-Ferrocenyl-3-((3-iodophenyl)amino)propan-1-ol (4b). Yellow oil. Yield 98\%. ${ }^{1} \mathrm{H}$ NMR $\left(200 \mathrm{MHz}, \mathrm{CDCl}_{3}\right) \delta=7.03-$ $6.77\left(\mathrm{~m}, 3 \mathrm{H}, 3 \times \mathrm{CH}_{\mathrm{Ar}}\right), 6.56-6.45\left(\mathrm{~m}, 1 \mathrm{H}, \mathrm{CH}_{\mathrm{Ar}}\right), 4.55-4.39$ $\left(\mathrm{m}, 1 \mathrm{H}, \mathrm{CHOHCH}_{2} \mathrm{CH}_{2} \mathrm{NH}\right), 4.26-4.07\left(\mathrm{~m}, 9 \mathrm{H}, 9 \times \mathrm{CH}_{\mathrm{Cp}}\right.$ *overlapped signals), $3.26-3.09$ (m, $2 \mathrm{H}, \mathrm{CHOHCH}_{2} \mathrm{CH}_{2} \mathrm{NH}$ ), $2.38-2.16\left(\mathrm{~m}, 1 \mathrm{H}, \mathrm{CHOHCH}_{2} \mathrm{CH}_{2} \mathrm{NH}\right), 2.07-1.75(\mathrm{~m}, 2 \mathrm{H}$, $\left.\mathrm{CHOHCH}_{2} \mathrm{CH}_{2} \mathrm{NH}\right) .{ }^{13} \mathrm{C} \mathrm{NMR}\left(50 \mathrm{MHz}, \mathrm{CDCl}_{3}\right) \delta=149.4$ $\left(\mathrm{C}_{\mathrm{Ar}}\right), 130.5\left(\mathrm{C}_{\mathrm{Ar}}\right), 125.7\left(\mathrm{C}_{\mathrm{Ar}}\right), 121.1\left(\mathrm{C}_{\mathrm{Ar}}\right), 112.0\left(\mathrm{C}_{\mathrm{Ar}}\right), 95.2$ $\left(\mathrm{C}_{\mathrm{Ar}}\right), 93.1\left(\mathrm{C}_{\mathrm{Cp}}\right), 68.4(\underline{\mathrm{CHOH}}), 68.2\left(5 \times \mathrm{C}_{\mathrm{Cp}}\right), 67.9\left(\mathrm{C}_{\mathrm{Cp}}\right), 67.9$ $\left(\mathrm{C}_{\mathrm{Cp}}\right), 66.6\left(\mathrm{C}_{\mathrm{Cp}}\right), 65.4\left(\mathrm{C}_{\mathrm{Cp}}\right), 40.9\left(\mathrm{CHOHCH}_{2} \mathrm{CH}_{2} \mathrm{NH}\right), 36.6$ 
$\left(\mathrm{CHOHCH}_{2} \mathrm{CH}_{2} \mathrm{NH}\right)$. IR $\left(\mathrm{ATR}, \mathrm{cm}^{-1}\right): v=3290(\mathrm{~N}-\mathrm{H}) \mathrm{cm}^{-1} ; v=$ $3080(\mathrm{O}-\mathrm{H}) \mathrm{cm}^{-1}$. Anal. Calc. for $\mathrm{C}_{19} \mathrm{H}_{20} \mathrm{FeINO}: \mathrm{C}, 49.49 ; \mathrm{H}$, 4.37; Fe, 12.11; I, 27.52; N, 3.04; O, 3.47. Found: C, 49.44, H, $4.41, \mathrm{~N}, 3.01 \%$.

1-Ferrocenyl-3-((4-iodophenyl)amino)propan-1-ol (4c). Yellow solid; mp $80{ }^{\circ} \mathrm{C}$ Yield $97 \% .{ }^{1} \mathrm{H}$ NMR $\left(200 \mathrm{MHz}, \mathrm{CDCl}_{3}\right)$ $\delta=7.39\left(\mathrm{~d}, J=8.6 \mathrm{~Hz}, 2 \mathrm{H}, 2 \times \mathrm{CH}_{\mathrm{Ar}}\right), 6.37(\mathrm{~d}, J=8.6 \mathrm{~Hz}, 2 \mathrm{H}, 2$ $\left.\times \mathrm{CH}_{\mathrm{Ar}}\right), 4.55-4.41\left(\mathrm{~m}, 1 \mathrm{H}, \mathrm{CHOHCH} \mathrm{CH}_{2} \mathrm{NH}\right), 4.29-4.03$ (m, $9 \mathrm{H}, 9 \times \mathrm{CH}_{\mathrm{Cp}}$ *overlapped signals), $3.33-3.07(\mathrm{~m}, 3 \mathrm{H}, 2 \mathrm{H}$, $\mathrm{CHOHCH}_{2} \mathrm{CH}_{2} \mathrm{NH}$ and $1 \mathrm{H}, \mathrm{CHOHCH}{ }_{2} \mathrm{CH}_{2} \mathrm{NH} *$ overlapped signals), $2.08-1.80\left(\mathrm{~m}, 2 \mathrm{H}, \mathrm{CHOHC} \underline{H}_{2} \mathrm{CH}_{2} \mathrm{NH}\right) .{ }^{13} \mathrm{C} \mathrm{NMR}(50$ $\left.\mathrm{MHz}, \mathrm{CDCl}_{3}\right) \delta=147.9\left(\mathrm{C}_{\mathrm{Ar}}\right), 135.5\left(\mathrm{C}_{\mathrm{Ar}}\right), 115.0\left(\mathrm{C}_{\mathrm{Ar}}\right), 93.5$ $\left(\mathrm{C}_{\mathrm{Ar}}\right), 85.2\left(\mathrm{C}_{\mathrm{Cp}_{\mathrm{p}}}\right), 68.6(\underline{\mathrm{C}} \mathrm{HOH}), 68.3\left(5 \times \mathrm{C}_{\mathrm{Cp}}\right), 68.1\left(\mathrm{C}_{\mathrm{Cp}}\right), 66.8$ $\left(\mathrm{C}_{\mathrm{Cp}}\right), 66.7\left(\mathrm{C}_{\mathrm{Cp}}\right), 65.3\left(\mathrm{C}_{\mathrm{Cp}}\right), 41.2\left(\mathrm{CHOHCH}_{2} \mathrm{CH}_{2} \mathrm{NH}\right), 36.9$ $\left(\mathrm{CHOHCH}_{2} \mathrm{CH}_{2} \mathrm{NH}\right)$. IR (ATR, $\left.\mathrm{cm}^{-1}\right): v=3300(\mathrm{~N}-\mathrm{H}) \mathrm{cm}^{-1} ; v=$ $3080(\mathrm{O}-\mathrm{H}) \mathrm{cm}^{-1}$. Anal. Calc. for $\mathrm{C}_{19} \mathrm{H}_{20} \mathrm{FeINO}$ : C, 49.49; $\mathrm{H}$, 4.37; Fe, 12.11; I, 27.52; N, 3.04; O, 3.47. Found: C, 49.53, H, $4.33, \mathrm{~N}, 3.08 \%$.

Synthesis of 1-aryl-4-ferrocenyl-3-phenyltetrahydropyrimidin2(1H)-ones (5a-c)

Ureas 5a-c were synthesized by the known procedure (Minić et al., 2015; Minić et al., 2020a). Spectral data of compounds 5a-c follow.

\section{4-(Ferrocenyl)-1-(2-iodophenyl)-3-phenyltetrahydro-}

pyrimidin-2(1H)-one $(5 \boldsymbol{a})$. **For example $5 \boldsymbol{a}$ we obtained a mixture of two atropoisomers (in the text below labeled as $\mathbf{5 a}+\mathbf{5 a}$ '), therefore, most of the signals in the NMR $\left({ }^{1} \mathrm{H}\right.$ NMR and ${ }^{13} \mathrm{C}$ NMR) spectra were double, which was in agreement with our previous studies (Minić et al., 2015; Minić et al., 2020).

Yellow oil. Yield 47\%. Isomers ratio 5a : 5a' $=59.5: 40.5$ and it has been calculated based on ${ }^{1} \mathrm{H}$ NMR spectra. ${ }^{1} \mathrm{H}$ NMR $\left(400 \mathrm{MHz}, \mathrm{CDCl}_{3}\right) \delta=7.91-7.84\left(\mathrm{~m}, 2 \mathrm{H}, 2 \times \mathrm{CH}_{\mathrm{Ar}}\right), 7.55(\mathrm{dt}, J$ $\left.=8.7,1.7 \mathrm{~Hz}, 3 \mathrm{H}, 3 \times \mathrm{CH}_{\mathrm{Ar}}\right), 7.49(\mathrm{dt}, J=6.4,1.3 \mathrm{~Hz}, 3 \mathrm{H}, 3 \times$ $\left.\mathrm{CH}_{\mathrm{Ar}}\right), 7.45-7.17\left(\mathrm{~m}, 8 \mathrm{H}, 8 \times \mathrm{CH}_{\mathrm{Ar}}\right), 7.09-6.95(\mathrm{~m}, 2 \mathrm{H}, 2 \times$ $\mathrm{CH}_{\mathrm{Ar}}$ ), 5.28 (pseudo t, $J=3.7 \mathrm{~Hz}, 1 \mathrm{H}, \mathrm{NCHCH} \mathrm{CH}_{2} \mathrm{~N}$ ), 5.22 (pseudo t, $J=3.7 \mathrm{~Hz}, 1 \mathrm{H}, \mathrm{NC}^{\mathrm{HCH}} \mathrm{CH}_{2} \mathrm{~N}$ ), $4.51(\mathrm{dt}, J=2.4,1.2$ $\left.\mathrm{Hz}, 1 \mathrm{H}, \mathrm{CH}_{\mathrm{Cp}}\right), 4.36\left(\mathrm{dt}, J=2.4,1.3 \mathrm{~Hz}, 1 \mathrm{H}, \mathrm{CH}_{\mathrm{Cp}}\right), 4.22-4.12$ $\left(\mathrm{m}, 3 \mathrm{H}, 3 \times \mathrm{CH}_{\mathrm{Cp}}\right), 4.11-4.09\left(\mathrm{~m}, 3 \mathrm{H}, 3 \times \mathrm{CH}_{\mathrm{Cp}}\right), 3.78(\mathrm{~s}, 5 \mathrm{H}, 5$ $\left.\times \mathrm{CH}_{\mathrm{Cp}}\right), 3.77\left(\mathrm{~s}, 5 \mathrm{H}, 5 \times \mathrm{CH}_{\mathrm{Cp}}\right), 3.63-3.58(\mathrm{~m}, 2 \mathrm{H}$, $\left.\mathrm{NCHC}_{2} \mathrm{CH}_{2} \mathrm{~N}\right), 3.45-3.31\left(\mathrm{~m}, 2 \mathrm{H}, \mathrm{NCHC}_{2} \mathrm{CH}_{2} \mathrm{~N}\right), 2.82-$ $2.59\left(\mathrm{~m}, 2 \mathrm{H}, \quad \mathrm{NCHCH}_{2} \mathrm{CH}_{2} \mathrm{~N}\right), 2.34-2.16(\mathrm{~m}, 2 \mathrm{H}$, $\left.\mathrm{NCHCH}_{2} \mathrm{CH}_{2} \mathrm{~N}\right) .{ }^{13} \mathrm{C}$ NMR $\left(101 \mathrm{MHz}, \mathrm{CDCl}_{3}\right) \delta=154.0$ $(\mathrm{N} \underline{\mathrm{CON}}), 153.3(\mathrm{~N} \underline{\mathrm{CON}}), 146.4\left(\mathrm{C}_{\mathrm{Ar}}\right), 145.8\left(\mathrm{C}_{\mathrm{Ar}}\right), 143.9\left(\mathrm{C}_{\mathrm{Ar}}\right)$, $143.5\left(\mathrm{C}_{\mathrm{Ar}}\right), 143.3\left(\mathrm{C}_{\mathrm{Ar}}\right), 143.2\left(\mathrm{C}_{\mathrm{Ar}}\right), 129.8\left(\mathrm{C}_{\mathrm{Ar}}\right), 129.5\left(\mathrm{C}_{\mathrm{Ar}}\right)$, $129.4\left(\mathrm{C}_{\mathrm{Ar}}\right), 129.3\left(\mathrm{C}_{\mathrm{Ar}}\right), 128.8\left(\mathrm{C}_{\mathrm{Ar}}\right), 128.6\left(\mathrm{C}_{\mathrm{Ar}}\right), 128.4\left(\mathrm{C}_{\mathrm{Ar}}\right)$, $128.1\left(\mathrm{C}_{\mathrm{Ar}}\right), 126.3\left(\mathrm{C}_{\mathrm{Ar}}\right), 126.2\left(\mathrm{C}_{\mathrm{Ar}}\right), 126.0\left(\mathrm{C}_{\mathrm{Ar}}\right), 125.6\left(\mathrm{C}_{\mathrm{Ar}}\right)$, $100.3\left(\mathrm{C}_{\mathrm{Ar}}\right), 99.4\left(\mathrm{C}_{\mathrm{Ar}}\right), 88.9\left(\mathrm{C}_{\mathrm{Cp}}\right), 88.8\left(\mathrm{C}_{\mathrm{Cp}}\right), 69.9\left(5 \times \mathrm{C}_{\mathrm{Cp}}\right)$, $68.9\left(5 \times \mathrm{C}_{\mathrm{Cp}_{\mathrm{p}}}\right), 68.8\left(\mathrm{C}_{\mathrm{Cp}}\right), 68.5\left(\mathrm{C}_{\mathrm{Cp}_{\mathrm{p}}}\right), 66.9\left(\mathrm{C}_{\mathrm{Cp}}\right), 66.8\left(\mathrm{C}_{\mathrm{Cp}_{\mathrm{p}}}\right)$, $66.6 \quad\left(\mathrm{C}_{\mathrm{Cp}_{\mathrm{p}}}\right), \quad 64.5 \quad\left(\mathrm{C}_{\mathrm{Cp}_{\mathrm{p}}}\right), \quad 64.4 \quad\left(\mathrm{C}_{\mathrm{Cp}_{\mathrm{p}}}\right), \quad 64.4 \quad\left(\mathrm{C}_{\mathrm{Cp}}\right), \quad 58.8$ $\left(\mathrm{N}^{2} \mathrm{HCH}_{2} \mathrm{CH}_{2} \mathrm{~N}\right), 58.6\left(\mathrm{~N}^{\mathrm{CHCH}} \mathrm{H}_{2} \mathrm{CH}_{2} \mathrm{~N}\right), 45.9\left(\mathrm{NCHCH}_{2} \mathrm{CH}_{2} \mathrm{~N}\right)$, 45.8 $\left(\mathrm{NCHCH}_{2} \underline{\mathrm{C}} \mathrm{H}_{2} \mathrm{~N}\right)$

\begin{abstract}
31.1
\end{abstract}
$\left(\mathrm{NCHCH}_{2} \mathrm{CH}_{2} \mathrm{~N}\right)$,
$\left(\mathrm{NCHCH}_{2} \mathrm{CH}_{2} \mathrm{~N}\right)$. IR $\left(\mathrm{ATR}, \mathrm{cm}^{-1}\right): v=1650(\mathrm{NCON}) \mathrm{cm}^{-1}$. Anal. Calc. for $\mathrm{C}_{26} \mathrm{H}_{23} \mathrm{FeIN}{ }_{2} \mathrm{O}: \mathrm{C}, 55.54 ; \mathrm{H}, 4.12 ; \mathrm{Fe}, 9.93 ; \mathrm{I}, 22.57 ; \mathrm{N}$, 4.98; O, 2.85. Found: C, 55.60; H, 4.07; N, 4.94\%.

\section{4-(Ferrocenyl)-1-(3-iodophenyl)-3-phenyltetrahydro-}

pyrimidin-2(1H)-one $(5 \boldsymbol{b})$. Yellow solid; mp $103{ }^{\circ} \mathrm{C}$. Yield $66 \%$. ${ }^{1} \mathrm{H}$ NMR $\left(400 \mathrm{MHz}, \mathrm{CDCl}_{3}\right) \delta=7.73\left(\mathrm{t}, J=1.9 \mathrm{~Hz}, 1 \mathrm{H}, \mathrm{CH}_{\mathrm{Ar}}\right)$, $7.52-7.40\left(\mathrm{~m}, 5 \mathrm{H}, 5 \times \mathrm{CH}_{\mathrm{Ar}}\right), 7.31(\mathrm{ddd}, J=8.1,2.1,1.0 \mathrm{~Hz}$, $\left.1 \mathrm{H}, \mathrm{CH}_{\mathrm{Ar}}\right), 7.28-7.23\left(\mathrm{~m}, 1 \mathrm{H}, \mathrm{CH}_{\mathrm{Ar}}\right), 7.05(\mathrm{t}, J=8.0 \mathrm{~Hz}, 1 \mathrm{H}$, $\mathrm{CH}_{\mathrm{Ar}}$ ), 5.20 (pseudo t, $J=3.9 \mathrm{~Hz}, 1 \mathrm{H}, \mathrm{NC} \underline{\mathrm{HCH}} \mathrm{CH}_{2} \mathrm{~N}$ ), $4.22-$ $4.18\left(\mathrm{~m}, 2 \mathrm{H}, 2 \times \mathrm{CH}_{\mathrm{Cp}}\right), 4.16-4.13\left(\mathrm{~m}, 1 \mathrm{H}, \mathrm{CH}_{\mathrm{Cp}}\right), 4.07(\mathrm{dt}, J=$ $\left.2.5,1.3 \mathrm{~Hz}, 1 \mathrm{H}, \mathrm{CH}_{\mathrm{Cp}}\right), 3.77\left(\mathrm{~s}, 5 \mathrm{H}, 5 \times \mathrm{CH}_{\mathrm{Cp}}\right), 3.65(\mathrm{td}, J=$ $\left.11.6, \quad 3.9 \mathrm{~Hz}, \quad 1 \mathrm{H}, \quad \mathrm{NCHCH}_{2} \mathrm{CH}_{2} \mathrm{~N}\right), \quad 3.53(\mathrm{~m}, \quad 1 \mathrm{H}$, $\mathrm{NCHCH}_{2} \mathrm{CH}_{2} \mathrm{~N}$ ), $2.68-2.57\left(\mathrm{~m}, 1 \mathrm{H}, \mathrm{NCHCH}_{2} \mathrm{CH}_{2} \mathrm{~N}\right), 2.25-$ $2.17\left(\mathrm{~m}, 1 \mathrm{H}, \mathrm{NCHCH}_{2} \mathrm{CH}_{2} \mathrm{~N}\right) \cdot{ }^{13} \mathrm{C} \mathrm{NMR}\left(101 \mathrm{MHz}, \mathrm{CDCl}_{3}\right) \delta=$ $153.6(\mathrm{NCON}), 145.0\left(\mathrm{C}_{\mathrm{Ar}}\right), 143.2\left(\mathrm{C}_{\mathrm{Ar}}\right), 134.6\left(\mathrm{C}_{\mathrm{Ar}}\right), 134.3$ $\left(\mathrm{C}_{\mathrm{Ar}}\right), 129.9\left(\mathrm{C}_{\mathrm{Ar}}\right), 128.5\left(\mathrm{C}_{\mathrm{Ar}}\right), 128.1\left(\mathrm{C}_{\mathrm{Ar}}\right), 126.4\left(\mathrm{C}_{\mathrm{Ar}}\right), 124.9$ $\left(\mathrm{C}_{\mathrm{Ar}}\right), 93.4\left(\mathrm{C}_{\mathrm{Ar}}\right), 88.6\left(\mathrm{C}_{\mathrm{Cp}_{\mathrm{p}}}\right), 68.8\left(5 \times \mathrm{C}_{\mathrm{Cp}}\right), 68.4\left(\mathrm{C}_{\mathrm{Cp}_{\mathrm{p}}}\right), 68.2$ $\left(\mathrm{C}_{\mathrm{Cp}}\right), 67.0\left(\mathrm{C}_{\mathrm{Cp}}\right), 64.4\left(\mathrm{C}_{\mathrm{Cp}}\right), 58.6\left(\mathrm{~N}^{\mathrm{CHCHCH}} \mathrm{CH}_{2} \mathrm{~N}\right), 45.6$ $\left(\mathrm{NCHCH}_{2} \mathrm{CH}_{2} \mathrm{~N}\right), 31.0\left(\mathrm{NCHCH}_{2} \mathrm{CH}_{2} \mathrm{~N}\right)$. IR $\left(\mathrm{ATR}, \mathrm{cm}^{-1}\right): v=$ 1640 (NCON) cm ${ }^{-1}$. Anal. Calc. for $\mathrm{C}_{26} \mathrm{H}_{23} \mathrm{FeIN}_{2} \mathrm{O}: \mathrm{C}, 55.54 ; \mathrm{H}$, 4.12; Fe, 9.93; I, 22.57; N, 4.98; O, 2.85. Found: C, 55.59; H, $4.08 ; \mathrm{N}, 4.93 \%$.

\section{4-(Ferrocenyl)-1-(4-iodophenyl)-3-phenyltetrahydro-}

pyrimidin-2(1H)-one $(5 \mathrm{c})$. Yellow solid; mp $92{ }^{\circ} \mathrm{C}$. Yield $74 \%$. ${ }^{1} \mathrm{H}$ NMR $\left(400 \mathrm{MHz}, \mathrm{CDCl}_{3}\right) \delta=7.63-7.58\left(\mathrm{~m}, 2 \mathrm{H}, 2 \times \mathrm{CH}_{\mathrm{Ar}}\right)$, $7.46-7.36\left(\mathrm{~m}, 4 \mathrm{H}, 4 \times \mathrm{CH}_{\mathrm{Ar}}\right), 7.24-7.19\left(\mathrm{~m}, 1 \mathrm{H}, \mathrm{CH}_{\mathrm{Ar}}\right), 7.10-$ $7.05\left(\mathrm{~m}, 2 \mathrm{H}, 2 \times \mathrm{CH}_{\mathrm{Ar}}\right), 5.17$ (pseudo $\mathrm{t}, J=3.9 \mathrm{~Hz}, 1 \mathrm{H}$, $\left.\mathrm{NC}^{\mathrm{H} C H} \mathrm{CH}_{2} \mathrm{~N}\right), 4.19-4.14\left(\mathrm{~m}, 2 \mathrm{H}, 2 \times \mathrm{CH}_{\mathrm{Cp}}\right), 4.10(\mathrm{td}, J=$ $\left.2.4,1.3 \mathrm{~Hz}, 1 \mathrm{H}, \mathrm{CH}_{\mathrm{Cp}}\right), 4.04\left(\mathrm{dt}, J=2.5,1.3 \mathrm{~Hz}, 1 \mathrm{H}, \mathrm{CH}_{\mathrm{Cp}}\right), 3.74$ $\left(\mathrm{s}, 5 \mathrm{H}, 5 \times \mathrm{CH}_{\mathrm{Cp}}\right), 3.61(\mathrm{td}, J=11.6,3.9 \mathrm{~Hz}, 1 \mathrm{H}$, $\left.\mathrm{NCHC}_{2} \mathrm{CH}_{2} \mathrm{~N}\right), 3.53-3.47\left(\mathrm{~m}, 1 \mathrm{H}, \mathrm{NCHC}_{2} \mathrm{CH}_{2} \mathrm{~N}\right), 2.65-$ $2.54\left(\mathrm{~m}, \quad 1 \mathrm{H}, \quad \mathrm{NCHCH}_{2} \mathrm{CH}_{2} \mathrm{~N}\right), 2.21-2.14(\mathrm{~m}, \quad 1 \mathrm{H}$, $\left.\mathrm{NCHCH}_{2} \mathrm{CH}_{2} \mathrm{~N}\right) .{ }^{13} \mathrm{C}$ NMR $\left(101 \mathrm{MHz}, \mathrm{CDCl}_{3}\right) \delta=153.7$ $(\mathrm{NCON}), 143.7\left(\mathrm{C}_{\mathrm{Ar}}\right), 143.2\left(\mathrm{C}_{\mathrm{Ar}}\right), 137.5\left(\mathrm{C}_{\mathrm{Ar}}\right), 128.5\left(\mathrm{C}_{\mathrm{Ar}}\right)$, $128.0\left(\mathrm{C}_{\mathrm{Ar}}\right), 127.6\left(\mathrm{C}_{\mathrm{Ar}}\right), 126.3\left(\mathrm{C}_{\mathrm{Ar}}\right), 89.6\left(\mathrm{C}_{\mathrm{Ar}}\right), 88.6\left(\mathrm{C}_{\mathrm{Cp}_{\mathrm{p}}}\right), 68.8$ $\left(5 \times \mathrm{C}_{\mathrm{C}_{\mathrm{p}}}\right), 68.4\left(\mathrm{C}_{\mathrm{Cp}}\right), 68.2\left(\mathrm{C}_{\mathrm{Cp}}\right), 66.9\left(\mathrm{C}_{\mathrm{Cp}}\right), 64.4\left(\mathrm{C}_{\mathrm{Cp}}\right), 58.6$ $\left(\mathrm{NCHCH}_{2} \mathrm{CH}_{2} \mathrm{~N}\right), 45.5\left(\mathrm{NCHCH}_{2} \mathrm{CH}_{2} \mathrm{~N}\right), 31.0\left(\mathrm{NCHCH}_{2} \mathrm{CH}_{2} \mathrm{~N}\right)$. IR $\left(\right.$ ATR, $\left.\mathrm{cm}^{-1}\right): v=1645(\mathrm{NCON}) \mathrm{cm}^{-1}$. Anal. Calc. for $\mathrm{C}_{26} \mathrm{H}_{23} \mathrm{FeIN}{ }_{2} \mathrm{O}: \mathrm{C}, 55.54 ; \mathrm{H}, 4.12 ; \mathrm{Fe}, 9.93 ; \mathrm{I}, 22.57 ; \mathrm{N}, 4.98 ; \mathrm{O}$, 2.85. Found: C, 55.58; H, 4.07; N, $4.91 \%$.

\section{RESULTS AND DISCUSSION}

\section{Synthesis}

Within the first phase of this synthesis, we made sure to come up with an appropriate method for the synthesis of 3-(arylamino)-1-ferrocenylproran-1-ones. Our team have already designed and optimized reaction conditions for the synthesis of 3-arylamino-1-ferrocenylpropan-1-ones. On this occasion, which took place a few years ago, we managed to obtain high yields of the said compounds, and these proved to be both biological agents, as well as exquisite starting materials which can be used 
for further chemical modifications (Damljanović et al., 2011; Pejović et al., 2012a; Pejović et al., 2012b; Minić et al., 2015; Minić et al., 2017; Minić et al., 2018; Minić et al., 2019; Minić et al., 2020a). In this manner, we adapted the protocol to make it suitable for the synthesis of target compounds through the usage of iodoaniline as the arylamino moiety (see Scheme 1). After setting the reaction, we were delighted to see that under these conditions we were indeed able to successfully synthesize 2ferrocenoyl ethyl aryl amines (3a-c) in the yields which were satisfactory (see Scheme 1 and Scheme 2).

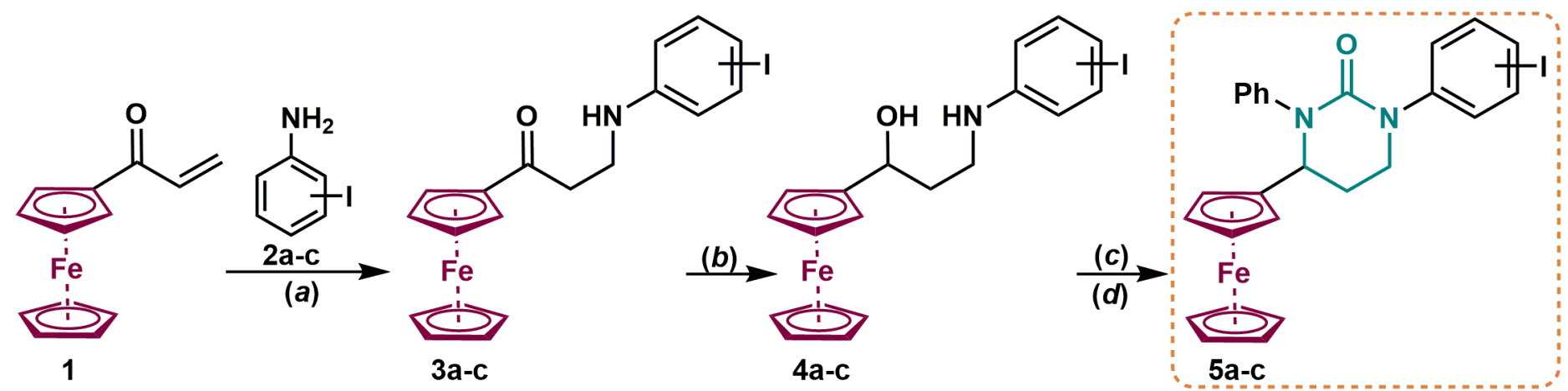

Scheme 1. Synthesis of novel Fc-containing derivatives $(\mathbf{3}, \mathbf{4}$ and 5). Reagents and conditions: (a) 1 (1 mmol), iodoaniline (2 mmol), montmorillonite K-10 (100 mg), ultrasound irradiation 1 h, r.t.; (b) 3a-c (1 mmol), $\mathrm{MeOH}(20 \mathrm{ml}), \mathrm{NaBH}_{4}(5 \mathrm{mmol}$, up to $190 \mathrm{mg})$, r.t.; $(c)$ 4a-c $(1 \mathrm{mmol}), \mathrm{PhNCO}(0.12 \mathrm{ml}, 1.1$ equivalents $)$, ultrasound irradiation $1 \mathrm{~h}$, r.t., then $(d) \mathrm{AcOH}(1$ $\mathrm{ml})$, ultrasound irradiation $1 \mathrm{~h}$, r.t.

The following step was to convert the obtained ketones 3ac into the 3-(arylamino)-1-ferrocenylproran-1-ols (4a-c). We utilized $\mathrm{NaBH}_{4}$ (5 equivalents) in $\mathrm{MeOH}$ for this reaction and we were also able to synthesize target compounds in a successful manner. This means that we managed to obtain exquisite yields (98\%), combined with a reduced reaction time and with the absence of sub products (see Scheme 1. and Scheme 2.). Our research team has previously reported on the method we utilized here (Minić et al., 2015; Minić et al., 2017; Minić et al., 2018; Minić et al., 2019; Minić et al., 2020b). It is essential to note that in organic chemistry $\gamma$-amino alcohols are generally regarded as versatile synthons. For that reason, the 4 a-c products we were able to obtain represent not only encouraging biological activity, but also remarkable starting material to be used for further synthesis of Fc-containing compounds. In this case, the focus is on an interest from a biological point of view (antibacterial, antimalarial, anti-inflammatory, antitumor etc.) (Minić et al., 2015; Minić et al., 2017; Minić et al., 2018; Minić et al., 2019; Minić et al., 2020a; Minić et al., 2020b; Pejović et al., 2015)

The last steps consisted of premising six-membered cyclic ureas 5a-c bearing ferrocenyl group (see Scheme 1). A method which we opted for in this synthetic transformation is both efficient and involves mild reaction conditions. The method was earlier reported on by our research team (Minić et al., 2015; Minić et al., 2020a). We started the investigation by ultrasonicating the mixture of 1,3-aminoalcohol (4a-c) and phenyl isocyanate $(0.12 \mathrm{ml}, 1.1 \mathrm{mmol})$. In the next step, we added $1 \mathrm{ml}$ of glacial acetic acid, and irradiation continued for additional $1.5 \mathrm{~h}$. Through the process of purification, we were able to isolate the desired heterocyclic scaffold $\mathbf{5}$, by using column chromatography in high purity ( $>95 \%$, confirmed by NMR) and good yield (see Scheme 2). It is important to note that in the case of the example $\mathbf{5 a}$ we were able to obtain the mixture of two atropoisomers $(\mathbf{5 a}+\mathbf{5 a}$ '; see Scheme 2). This is the result which corresponds to previous study by our team (Minić et al., 2020a) However, regretfully, we haven’t succeeded in separating these isomers.

Despite the fact that the reaction of 1,3-amino alcohols 4a-c with phenyl isocyanate and the following intramolecular cyclization in the presence of acetic acid to cyclic ureas $\mathbf{5 a - c}$ was carried out in "a one-pot" manner, we have previously established the existence of intermediates - the corresponding hydroxy ureas (6a-c) by carefully analyzing ${ }^{1} \mathrm{H}$ and ${ }^{13} \mathrm{C}$ NMR spectra (see Scheme 3) (Minić et al., 2015; Minić et al., 2020a).

In order to get a more comprehensive insight into the role of $\alpha$-ferrocenyl carbocation, we discussed the reaction mechanism. As demonstrated in Scheme 3, the beginning of the reaction is marked by the protonation of $\beta$-hydroxy ureas 6a-c by acetic acid and dehydration of the resulting oxonium ions II, eventually giving $\alpha$-ferrocenyl carbocations III (Wagner, G., \& Herrmann, R., 1995). Finally, a nucleophilic attack of the carbamide nitrogen on the positive centre of $\alpha$-ferrocenyl carbocations III forms cations IV, which yielded the target 1-aryl-4-ferrocenyl-3-phenyltetrahydropyrimidin$2(1 H)$-ones after deprotonations (5a-c). 

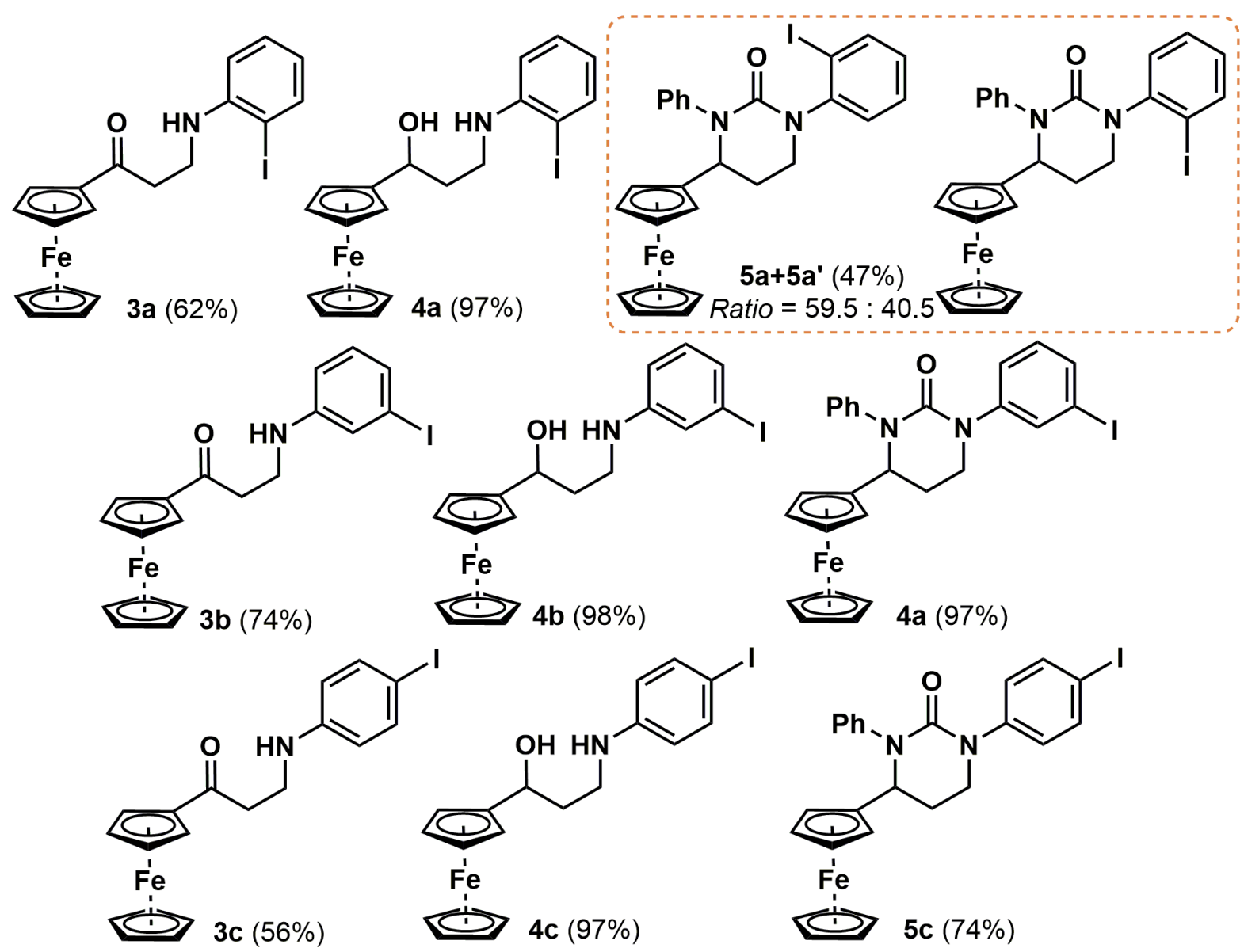

Scheme 2. An overview of newly prepared Fc-derivatives 3, 4 and 5.
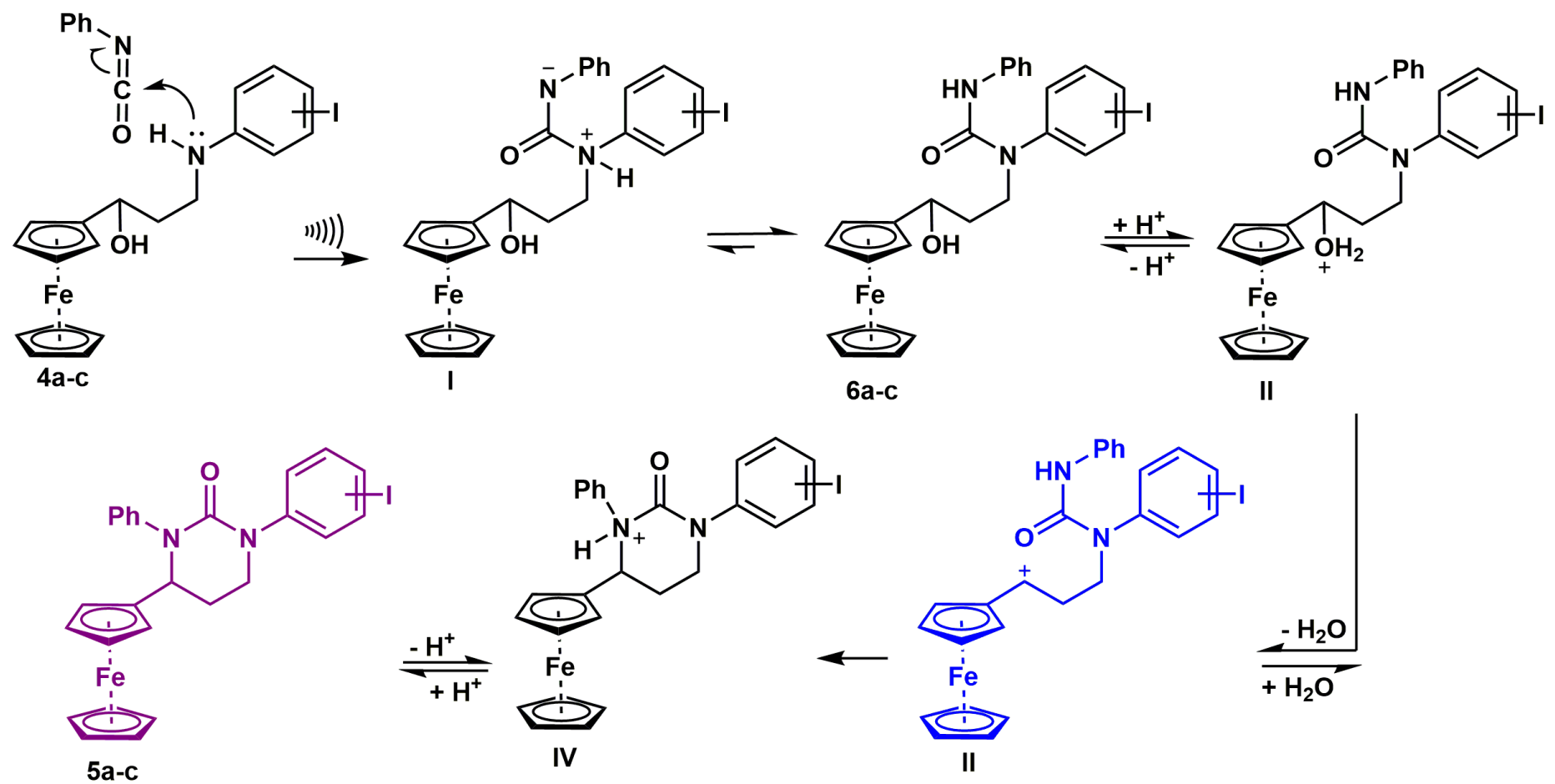

Scheme 3. A plausible reaction mechanism of the transformation of 1,3-amino alcohols 4a-c into 5a-c. 


\section{Spectral characterization}

In order to confirm structure of the newly prepared molecules $\mathbf{3}, \mathbf{4}$ and $\mathbf{5}$, we have performed a detailed characterization by standard spectroscopic techniques (IR, ${ }^{1} \mathrm{H}$ and ${ }^{13} \mathrm{C}$ NMR), as well as elemental analyses. We have found all spectral data to be entirely compatible to the projected structures (for more data see Experimental part).

Within the IR spectra of 3a-c, we have observed sharp, medium intensity absorption of $\mathrm{NH}$ stretching vibration below $3200 \mathrm{~cm}^{-1}$, thereby demonstrating that all $\mathrm{NH}$ groups participate in H-bonding interactions. The $\mathrm{CO}$ stretching vibration band of the 1'-ferrocene-carbonyl group seem to be at approximately $1655 \mathrm{~cm}^{-1}$. The ${ }^{1} \mathrm{H}$ and ${ }^{13} \mathrm{C}$ NMR spectra of compound 3a-c demonstrate all the signals (for aliphatic, amino, ferrocenyl and aromatic protons) normally foreseen for the projected composition.

Infrared spectra of the Fc-containing 1,3-amino alcohols 4a-c exhibit characteristic bands related to $\mathrm{N}-\mathrm{H}$ stretching vibrations approximately $3290 \mathrm{~cm}^{-1}$ and $\mathrm{O}-\mathrm{H}$ stretching vibrations approximately $3080 \mathrm{~cm}^{-1}$. Furthermore, the lack of signal for $\mathrm{C}=\mathrm{O}$ undeniably validates the occurrence of the reduction. The ${ }^{1} \mathrm{H}$ NMR spectra of $\mathbf{4 a - c}$ comprises the signals which are typical of the aliphatic, hydroxy, ferrocene, amino, and aromatic protons found in the expected regions. In the ${ }^{13} \mathrm{C} N \mathrm{NR}$ spectra of the $\mathbf{4 a - c}$, we can recognize the signals of the aliphatic, ferrocene and aromatic carbons in suitable regions. Additionally, the lack of signal which is associated with carbonyl group in ${ }^{13} \mathrm{C}$ NMR spectra of compound $\mathbf{4 a - c}$ and the presence of signal at $\sim 68.5 \mathrm{ppm}$, originated from $\mathrm{CHOH}$, unquestionably validates the projected product structure.

The IR spectra of the obtained compound 5a-c comprises projected bands carbonyl diamide group. In the ${ }^{1} \mathrm{H}$ NMR spectra of product mixture $\mathbf{5} \mathbf{a}+\mathbf{5} \mathbf{a}^{\prime}$ the signals typical of the aromatic, ferrocene, and aliphatic protons can be found in pairs in the projected regions. The corresponding signal for compounds $\mathbf{5 b}$ and $\mathbf{5 c}$ has been observed, as well. In the ${ }^{13} \mathrm{C}$ NMR spectra of 1aryl-4-ferrocenyl-3-phenyltetrahydropyrimidin-2(1H)-ones c) signals of the aliphatic, ferrocenyl, aromatic and carbonyl diamide carbons can be recognized in suitable regions (for example $\mathbf{5 a}+\mathbf{5} \mathbf{a}$ ' the majority of the signals can be recognized in pairs).

\section{CONCLUSION}

In conclusion, this paper presents the synthesis of three novel six-membered cyclic ureas bearing a ferrocenyl core starting from appropriate 1,3-amino alcohol. The protocol used is found to be practical, as well as convenient. Structures of all newly prepared compounds were undeniably validated using the standard spectroscopic techniques and elemental analyses. Finally, we find the synthesized compounds to be of potential interest for the bioactivity studies.

\section{ACKNOWLEDGMENTS}

This work was supported by the Ministry of Education, Science and Technological development of the Republic of Serbia (Agreement No. 451-03-68/2020-14/200122).

\section{REFERENCES}

Damljanović, I., Stevanović, D., Pejović, A., Vukićević, M., Novaković, S. B., Bogdanović, G. A., Mihajilov-Krstev, M. T., Radulović, N. \& Vukićević, R. D. 2011. Antibacterial 3(arylamino)-1-ferrocenylpropan-1-ones: Synthesis, spectral, electrochemical and structural characterization. Journal of Organometallic Chemistry, 696, pp. 3703-3713. doi.org/10.1016/j.jorganchem.2011.08.016

Domling, A., Wang, W., \& Wang, K., 2012. Chemistry and Biology Of Multicomponent Reactions. Chemistry Reviews, 112, pp. 3083-3135. https://doi.org/10.1021/cr100233r

Eicher, T., Hauptmann, S. \& Speicher, A. 2003. The Chemistry of Heterocycles: Structure, Reactions, Syntheses, and Applications, $2^{\text {nd }}$ ed., Wiiey-VCH Veriag, doi.10.1002/352760183X

Fernandez, M. \& Caballero, J. 2006. Modeling of activity of cyclic urea HIV-1 protease inhibitors using regularizedartificial neural networks, Bioorganic \& Medicinal Chemistry, $\quad 16, \quad$ pp. 280-294 https://doi.org/10.1016/j.bmc.2005.08.022

Hayashi, Y. 2016. Pot economy and one-pot synthesis. Chemical Science, 7, pp. 866-880. DOI: 10.1039/C5SC02913A

Kaneko, T., McMillen, W. \& Lynch, M. K. 2007. Synthesis and antibacterial activity of $\mathrm{C} 11, \mathrm{C} 12$-cyclic urea analogues of ketolides, Bioorganic \& Medicinal Chemistry Letters, 17, pp. 5013-5018. https://doi.org/10.1016/j.bmcl.2007.07.041

Kashawa, S. K., Kashawa, V., Mishra, P., Jain, N. K. \& Stables, J. P. 2009. Synthesis, anticonvulsant and CNS depressant activity of some new bioactive 1-(4-substituted-phenyl)-3-(4oxo-2-phenyl/ethyl-4H-quinazolin-3-yl)-urea, European Journal of Medicinal Chemistry, 44, pp. 4335-4343. https://doi.org/10.1016/j.ejmech.2009.05.008

Khan, S. A., Singh, N. \& Saleem, K. 2008. Synthesis, characterization and in vitro antibacterial activity of thiourea and urea derivatives of steroids, European Journal of Medicinal Chemistry, 43, pp. 2272-2277. https://doi.org/10.1016/j.ejmech.2007.12.012

Li, H. Q., Zhu, T. T., Yan, T., Luo, Y. \& Zhu, H. L. 2009. Design, synthesis and structure-activity relationships of antiproliferative 1,3-disubstituted urea derivatives, European Journal of Medicinal Chemistry, 44, pp. 453-459. https://doi.org/10.1016/j.ejmech.2008.04.011

Minić, A., Stevanović, D., Vukićević, M., Bogdanović, G. A., D'hooghe, M., Radulović, N. \& Vukićević, R. D. 2017. Synthesis of novel 4-ferrocenyl-1,2,3,4-tetrahydroquinolines and 4 -ferrocenylquinolines via $\alpha$-ferrocenyl carbenium ions as key intermediates. Tetrahedron, 73, pp 6268-6274. doi. 10.1016/j.tet.2017.09.014

Minić, A., Bugarinović, J., Ilić-Komatina, D., Bogdanović, G. A., Damljanović, I. \& Stevanović, D. 2018. Synthesis of novel ferrocene-containing 1,3-thiazinan-2-imines: One-pot reaction promoted by ultrasound irradiation. Tetrahedron Letters, 59, pp. 3499-3502. doi. 10.1016/j.tetlet.2018.08.029 
Minić, A., Bugarinović, J., Pešić, M. \& Ilić-Komatina, D. 2019. Novel 4-ferrocenyl-8-(phenylthio)-1,2,3,4-tetrahydroquinoline: design, synthesis and spectral characterization, University Thought - Publication in Natural Sciences, 9(1), pp. 38-44. DOI:10.5937/univtho9-20839

Minić, A., Stevanović, D., Damljanović, I., Pejović, A., Vukićević, M., Bogdanović, G. A., Radulović, N. \& Vukićević, R. D. 2015. Synthesis of ferrocene-containing sixmembered cyclic ureas via $\alpha$-ferrocenyl carbocations. RSC Advances, 5, pp. 24915-24919. doi. 10.1039/C5RA01383F

Minić, A., Novaković, S. B., Bogdanović, G. A., Bugarinović, J., Pešić, M., Todosijević, A., Ilić-Komatina, D., Damljanović, I. \& Stevanović, D. 2020a. Synthesis and structural characterizations of novel atropoisomeric ferrocenecontaining six-membered cyclic ureas, Polyhedron, $177 \mathrm{pp}$. 114316. https://doi.org/10.1016/j.poly.2019.114316

Minić, A., Van de Walle, T., Van Hecke, K., Combrinck, J., Smith, P. J., Chibale, K. \& D'hooghe, M. 2020b. Design and synthesis of novel ferrocene-quinoline conjugates and evaluation of their electrochemical and antiplasmodium properties, European Journal of Medicinal Chemistry, 187, pp. 111963. https://doi.org/10.1016/j.ejmech.2019.111963

Motokura, K., Fujita, N., Mori, K., Mizugaki, T., Ebitani, K. \& Kaneda, K. 2005. An Acidic Layered Clay Is Combined with A Basic Layered Clay for One-Pot Sequential Reactions. Journal of the American Chemical Society, 127, pp. 96749675. https://doi.org/10.1021/ja052386p

Nicolaou, K. C., Edmons, D. J. \& Bulger, P. G. 2006. Cascade Reactions in Total Synthesis. Angewandte Chemie International Edition, 45, pp. 7134-7186. https://doi.org/10.1002/anie.200601872

Pejović, A., Stevanović, D., Damljanović, I., Vukićević, M., Novaković, S. B., Bogdanović, G. A., Mihajilov-Krstev, M. T., Radulović, N. \& Vukićević, R. D. 2012a. Ultrasound-assisted synthesis of 3-(arylamino)-1-ferrocenylpropan-1-ones. Helvetica Chimica Acta, 95, pp. 1425-1441. doi.org/10.1002/hlca.201200009

Pejović, A., Damljanović, I., Stevanović, D., Vukićević, M., Novaković, S. B., Bogdanović, G. A., Radulović, N. \& Vukićević, R. D. 2012b. Antimicrobial ferrocene containing quinolinones: Synthesis, spectral, electrochemical and structural characterization of 2-ferrocenyl-2,3dihydroquinolin-4(1H)-one and its 6-chloro and 6-bromo derivatives, Polyhedron, 31, pp. 789-795. https://doi.org/10.1016/j.poly.2011.11.006

Pejović, A., Damljanović, I., Stevanović, D., Minić, A., Jovanović, J., Mihailović, V., Katanić, J. \& Bogdanović, G. A. 2017. Synthesis, characterization and antimicrobial activity of novel ferrocene containing quinolines: 2ferrocenyl-4-methoxyquinolines, 1-benzyl-2-ferrocenyl-2,3dihydroquinolin-4(1H)-ones and 1-benzyl-2-
ferrocenylquinolin-4(1H)-ones, Journal of Organometallic Chemistry, 846, pp. 6-17. http://dx.doi.org/10.1016/j.jorganchem.2017.05.051

Pejović, A., Minić, A., Bugarinović, J., Pešić, M., Damljanović, I., Stevanović, D., Mihailović, V., Katanić, J. \& Bogdanović, G. A. 2018a. Synthesis, characterization and antimicrobial activity of novel 3-ferrocenyl-2-pyrazolyl-1,3-thiazolidin-4ones, Polyhedron, 155, pp. 382-389. https://doi.org/10.1016/j.poly.2018.08.071

Pejović, A., Minić, A., Jovanović, J., Pešić, M., Ilić Komatina, D., Damljanović, I., Stevanović, D., Mihailović, V., Katanić, J. \& Bogdanović, G. A. 2018b. Synthesis, characterization, antioxidant and antimicrobial activity of novel 5-arylidene-2ferrocenyl-1,3-thiazolidin-4-ones, Journal of Organometallic Chemistry, $869, \quad$ pp. $1-10$ https://doi.org/10.1016/j.jorganchem.2018.05.014

Pejović, A., Danneels, B., Desmet, T., Cham, B. T., Nguyen, T., Radulović, N.S., Vukićević, R. D. \& D'hooghe, M. 2015. Synthesis and Antimicrobial/Cytotoxic Assessment of Ferrocenyl Oxazinanes, Oxazinan-2-ones, and Tetrahydropyrimidin-2-ones, Synlett, 26, pp. 1195-1200. doi. 10.1055/s-0034-1380348

Sarma, R., Sarmah, M. M., \& Prajapati, D., 2012a. MicrowavePromoted Catalyst- and Solvent-Free Aza-Diels-Alder Reaction of Aldimines with 6-[2-(Dimethylamino)vinyl]-1,3dimethyluracil. Journal of Organic Chemistry, 77, pp. 2018 2023. https://doi.org/10.1021/jo202346w

Sharma, A., Appukkuttan, P., \& Van der Eycken, E. V., 2012 b. Microwave-assisted synthesis of medium-sized heterocycles. Chemical Communications, 48, pp. 1623-1637. DOI: 10.1039/C1CC15238F

Taylor, A. P., Robinson, R. P., Fobian, Y. M., Blakemore, D. C., Jones, L. H. \& Fadeyi, O. 2016. Modern advances in heterocyclic chemistry in drug discovery. Organic \& Biomolecular Chemistry, 14, pp. 6611-6637. doi.10.1039/C6OB00936K

Tale, R. T., Rodge, A. H., Hatnapure, G. D., \& Keche, A. P. 2011. The novel 3,4-dihydropyrimidin-2(1H)-one urea derivatives of $\mathrm{N}$-aryl urea: Synthesis, anti-inflammatory, antibacterial and antifungal activity evaluation, Bioorganic \& Medicinal Chemistry Letters, 21, pp. 4648-4651. https://doi.org/10.1016/j.bmcl.2011.03.062

Wagner, G., \& Herrmann, R., 1995. Chiral Ferrocene Derivatives, An Introduction, in Ferrocenes: Homogenous Catalysts, Organic Synthesis, Material Science, ed. A. Togni and T. Hayashi, VCH, Weinheim, pp. 175-178.

Yang, B., Tao, C., Shao, T., Gong, J., \& Che, C., 2016. One-pot synthesis of tetracyclic fused imidazo[1,2-a]pyridines via a three-component reaction. Beilstein Journal of Organic Chemistry, 12, pp 1487-1492. doi:10.3762/bjoc.12.145 